\title{
A new non-invasive estimation of the stenotic aortic valve area by pulsed Doppler mapping
}

\author{
COLETTE VEYRAT, * C GOURTCHIGLOUIAN, P DUMORA, G ABITBOL, \\ D SAINTE BEUVE, D KALMANSON
}

From the Service de Cardiologie, Fondation A de Rothschild, Paris, France

SUMMARY A new pulsed Doppler mapping technique has been used to measure the severity of aortic valve stenosis. The Doppler examination was performed at the site of the aortic orifice in the parasternal short axis echocardiographic view and the method was based on the detection of the area of systolic flow through the stenotic orifice. This area was derived by planimetry and the measurements obtained by the Doppler method were compared with the aortic valve area calcu $\frac{\epsilon}{\Phi}$ lated at catheterisation according to the Gorlin formula. The method was applicable in 41 of the 44 patients studied. The Doppler data were consistent with the haemodynamic measurements even in patients with decreased cardiac index.

It is concluded that this new application of the flow mapping procedure is reliable and is easilypo applied to adult patients with a wide range of clinical conditions.

Knowledge of the aortic valve area is important in the management of patients with aortic stenosis. In adults standard non-invasive procedures have failed to provide a variable that reliably predicts the aortic valve area. ${ }^{1-3}$ Doppler echocardiography would be expected to be the most appropriate of recent developments because it measures flow. Aortic stenosis can be measured by continuous wave Doppler. This method relies on the calculation of the pressure gradient across the orifice ${ }^{4-6}$ The shortcoming of this variable is that it is dependent on cardiac output. The semilunar stenotic area has been calculated by non-invasive measurement of both the pressure gradient and cardiac output in children. ${ }^{7}$ Non-invasive measurement of the cardiac output is feasible in adults, ${ }^{89}$ but is not yet routinely applicable particularly in elderly patients and in a recent attempt to calculate the aortic valve area with continuous wave Doppler in an adult population invasive measurement of cardiac output was still required. ${ }^{10} \mathrm{We}$ present a new non-invasive method of measuring aortic stenosis with pulsed Doppler. It is based on

Requests for reprints to Dr Colette Veyrat, Service de Cardiologie, Fondation A de Rothschild, 25-29 rue Manin, 75940 Paris Cedex 19, France.

*Chargé de Recherches au CNRS.

Accepted for publication 28 August 1986 flow mapping at the aortic orifice, which is used to delineate the aortic valve area. To assess th reliability of the new procedure we have compared it with results derived from catheterisation.

\section{Patients and methods}

We studied 44 consecutive patients $(21$ men and 23 women (mean age 58)) with aortic stenosis wh\& underwent left heart catheterisation. Forty two patients were in sinus rhythm and two were in atrial. fibrillation. The aetiology was congenital (2), rheuo matic (12), or degenerative with calcified aortie cusps (30). The stenosis was sometimes associate with regurgitation; this was graded as minimal in seven cases and as moderate in five other cases. The other associated abnormalities were mitral stenosif (6), or prosthesis (2), tricuspid regurgitation (7), caro diomyopathy (2), coronary heart disease (4), ant angiodysplasia (1). Twenty eight patients later ha\& surgery.

We also studied a control group of 12 patients men and 5 women (mean age 47 years)) with nळ aortic gradient at cardiac catheterisation. All but one were in sinus rhythm. Diagnoses were mitral steno미 sis (6), non-obstructive cardiomyopathy (3), coroo nary heart disease (2), and atrial septal defect. (1).

INVASIVE PROCEDURES

The diagnosis was assessed by means of left heare 
retrograde or transseptal catheterisation, or both, by standard techniques. The aortic valve area was calculated according to the Gorlin formula. ${ }^{11}$ Cardiac output was measured by the Fick method or thermodilution, or both. Supravalvar aortography was performed from a $30^{\circ}$ or $45^{\circ}$ right anterior oblique position. The severity of the regurgitation was assessed by qualitative criteria on a three point grading scale. $^{12}$

\section{DOPPLER ECHOCARDIOGRAPHY}

We used a $3 \mathrm{MHz}$ ATL 851 (Squibb Medical System, Bellevue, Washington, USA) mechanical real time scanner, which provided a $90^{\circ}$ sector and single gate steerable pulsed Doppler capability, with a tear drop shaped $(2 \times 4 \mathrm{~mm})$ sample volume. ${ }^{13}$ The Doppler output could be presented as an audible signal, a time interval histogram, and a real time frequency spectrum by means of fast Fourier transform analysers. These devices were either an Angioscan (Unigon Industries, Mount Vernon, NY, USA), or a Sonacolor (Carolina Medical Electronics, King, NC, USA). The principles and characteristics of the instruments have been described elsewhere. ${ }^{1415}$

We used two video monitors, one for real time scanning and the other for the Doppler display.

Hard copies of real time images were made on a 4633 Tektronics recorder. $M$ mode echocardiographic tracings, simultaneous electrocardiograms, phonocardiograms, and Doppler spectral displays were recorded on an Irex 101 (Irex, Mahwah, NJ, USA) multitrace recorder or on Polaroid films.

Subjects were generally studied in the left lateral supine position within a few days of catheterisation. The method of cross sectional Doppler echocardiographic examination of the heart ${ }^{16}$ that we used requires dynamic visualisation of the structure or chamber under investigation, location of the Doppler beam seen as a white line crossing the area of interest, and adjustment of the Doppler gate, seen as a white spot, to control the depth of the sample volume along that beam. The image is then frozen while the apparatus is automatically switched to the Doppler system. When the characteristic Doppler sound is heard, the recording is performed.

\section{EXAMINATION OF THE AORTIC VALVE ORIFICE (FIG 1A)}

We used the left parasternal short axis view to get a satisfactory image of the aortic orifice. Visualisation of part of the tips of the cusps indicated that the Doppler beam was located in the plane required. In
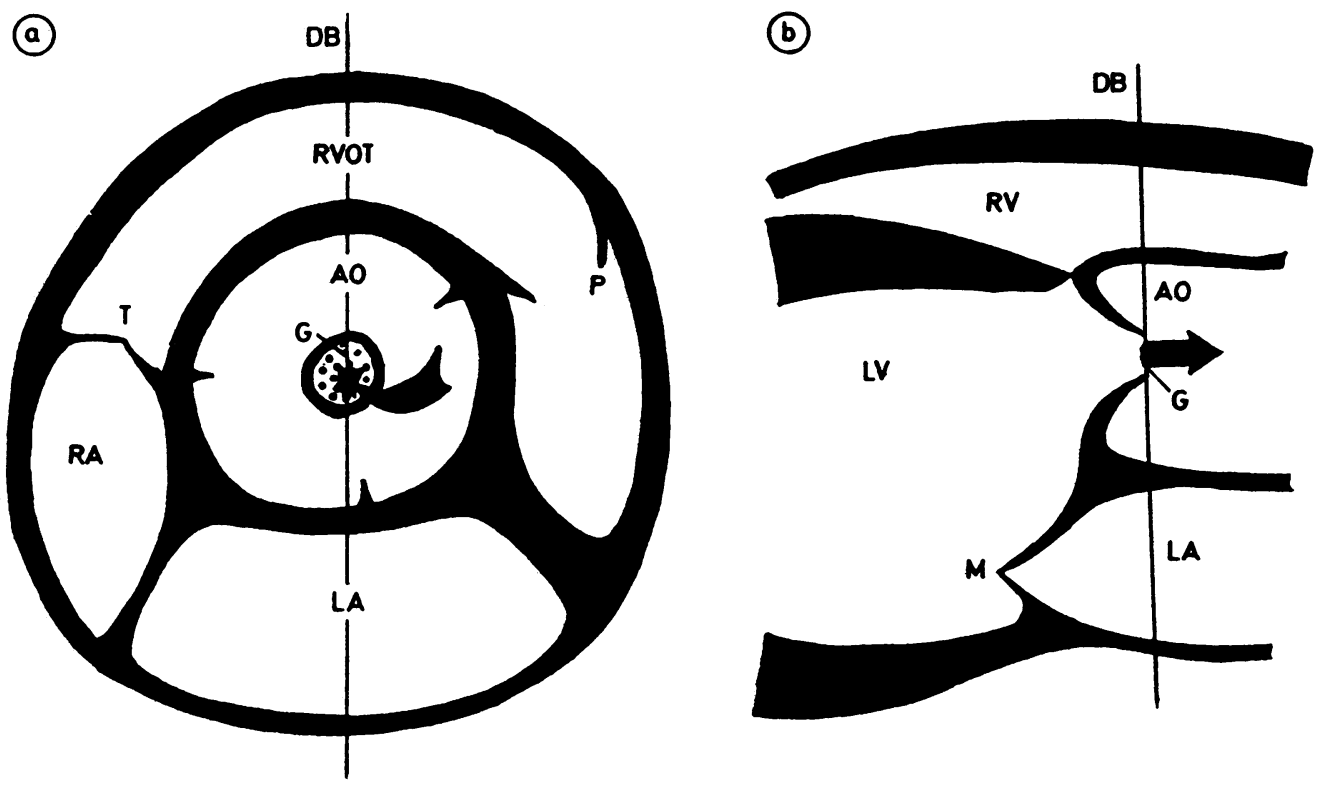

Fig 1 Schematic representation of the mapping procedure used for measuring the stenotic aortic valve arca in aortic stenosis. (a) Mapping of the stenotic aortic valve area (dotted area) in short axis echocardiographic view. The Doppler beam (vertical line) transsects the aortic orifice and the Doppler gate is moved over the orifice to detect a systolic flow signal (arrow). The limits of the area are defined by to-and-fro scanning movements from the centre to the boundary edges. (b) Determination of the tips of the cusps by long axis examination. The Doppler beam is located beyond the cusps (vertical line), and the Doppler gate detects the area of systolic flow (arrow) along the anteroposterior axis of the aortic root. (AO, aortic orifice; $D B$, Doppler beam; $G$, Doppler gate; $L A$, left atrium; $L V$, left ventricle; $M$, mitral valve; $P$, pulmonary valve; $R A$, right atrium; $R V$, right ventricle; $R V O T$, right ventricle outflow tract; $T$, tricuspid valve. 
patients with important calcification the transducer was oriented slightly upwards. Once the cusps had been visualised the image was frozen in systole and the following procedure was performed. We carefully searched for a Doppler signal with maximum amplitude and duration occurring before the end of systole whether it was positive, negative, or both. When this signal was detected we performed to-andfro mapping of the area where it was recorded, from its centre to its boundary edges, along its anteroposterior, transverse, and oblique axes to obtain a measure of the aortic valve area. A sheet of transparent plastic was applied over the video monitor and the contour of the area was traced either during subsequent repeated manoeuvres or during a later review of the video tape. We took care to avoid parallax errors. We also mapped the entire aortic orifice using the same procedure to define the anatomical relations of the stenotic area to the orifice.

During the examination we paid particular attention to the following points. To minimise artefact due to motion of the aortic area during systole, the timing of early systole was noted when we determined the first location point of the mapping and this was maintained for the determination of all subsequent mapping points; to check that the site of the recording coincided exactly with the plane of the aortic orifice we crosschecked the anteroposterior measurement (in millimetres) obtained in the short axis view against that obtained in long axis view at the tip of the aortic cusps using the same Doppler criteria (fig 1b). If necessary, in the case of eccentric areas leading to eccentric flow signals, we used a manoeuvre derived from that proposed by Nishimura et al for the mitral apparatus, ${ }^{17}$ that is we used two complementary parallel long axis views transsecting the long axis of the heart on each side of the central long axis view.

Three sequences of systolic beats were analysed and averaged to determine the measurements. All patients were examined without knowledge of the results of the invasive procedures, which were performed by separate teams. The first 31 patients were investigated by two independent observers. Each videotape was reviewed by each observer within three weeks of the examination and there was no significance intraobserver discrepancy between the results.

The stenotic valve areas were measured by planimetry with a Hewlett Packard integrator 98134 A with a $1 \%$ error estimated from planimetry of test areas of given values. For the statistical study, each pair of values was displayed together with the line of equality, both for the aortic valve area and for the study of interobserver agreement. The variance of the paired measurements was also studied and the differences between the measurements obtained from both methods were plotted against the mea value of these measurements to study the agreemerft between the methods. ${ }^{18}$

\section{Results}

\section{CONTROLS}

An audiosignal of flow with smooth tone was hear $\frac{\widehat{\Phi}}{\Phi}$ over most of the aortic orifice in all patients. Th\% graphic display showed a net velocity vector away from the zero line in only five patients. Among the remaining patients the signal was too weak to geners్ ate such a deflection, and the graphic display consis? ted of an early and mid-systolic broadened spectrum between two transient velocity deflections due t\& cusp motion that was widely spread over the orifice with a harsher tone when approaching the boundar edges (fig 2).

PATIENTS WITH AORTIC STENOSIS (TABLE 1, FIGS 3-6)

Recordings were unsatisfactory in three patients because of poor signal to noise ratio. The Doppler

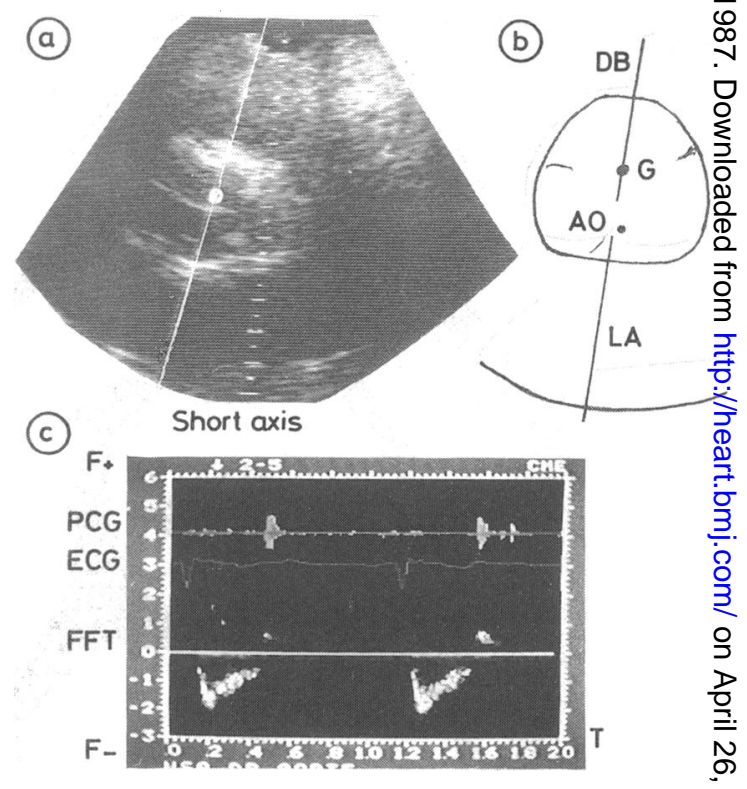

Fig 2 Normal aortic orifice. (a) Short axis scan view of thS aortic orifice with the Doppler gate shown in the centre of the orifice on the schematic drawing (b). (c) Pulsed Doppler recording of systolic flow obtained by fast Fourier transform spectral analysis. There is a systolic deflection away from thes zero line with a laminar spectrum. This signal was heard over most of the aortic area in this patient. In half the patients with normal aortic valves no clear deflection was seen. Instead there was an early systolic spectral broadening recorded over most of the aortic orifice. See legend to fig 1 for abbreviations. FFT, fast Fourier transform; ECG, electrocardiogram; PCG, phonocardiogram; $F+$ and $F-, \underset{<}{\sigma}$ positive and negative Doppler shifts; $T$, time axis. 


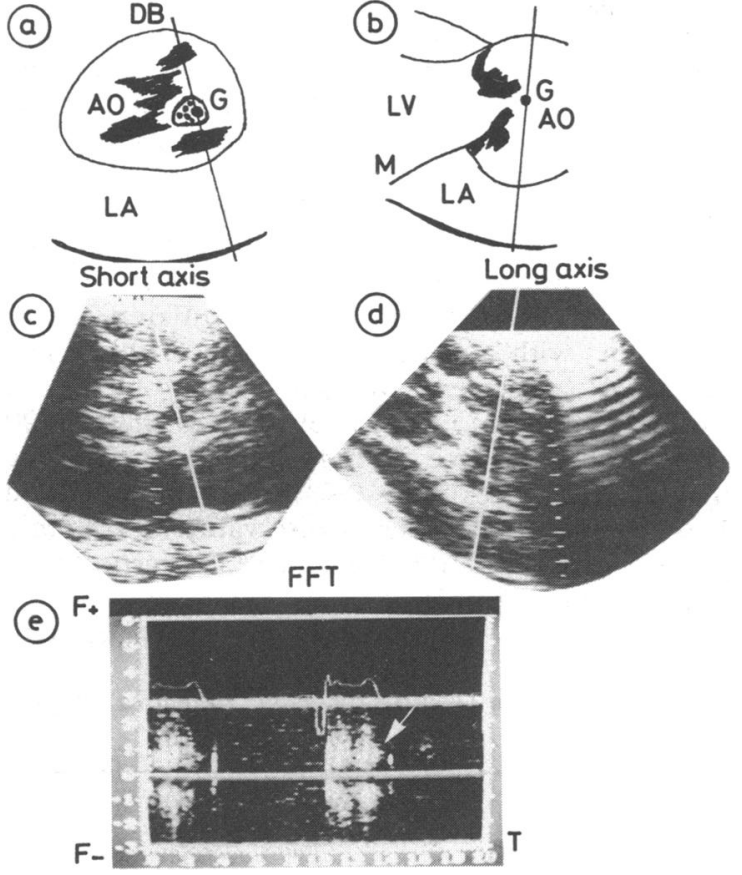

Fig 3 Patient with aortic stenosis. Drawings of the short axis (a) and the long axis (b) views of the aortic orifice. The gate is located at the site of the narrowing area (stippled area). Scan views of both planes in which the aortic orifice was visualised ( $c$ and d). (e) Real time Doppler recording of spectral analysis showing the systolic spectral anomalies (white arrow) peaking before end systole. No Doppler flow signal was recorded over the remaining orifice area. See figures 1 and 2 for abbreviations.

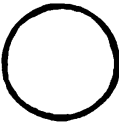

$56 \%$

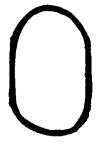

$12 \%$

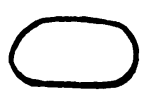

$14 \%$

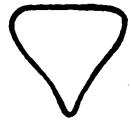

$10 \%$
$8 \%$

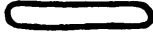

Fig 4 Various shapes of stenotic areas found by the flow mapping procedure. The frequency $(\%)$ of each type of stenotic area is indicated. Only three patients $(8 \%)$ had a slit shaped orifice. Two of them were operated upon and were found to have a Monckeberg disease.

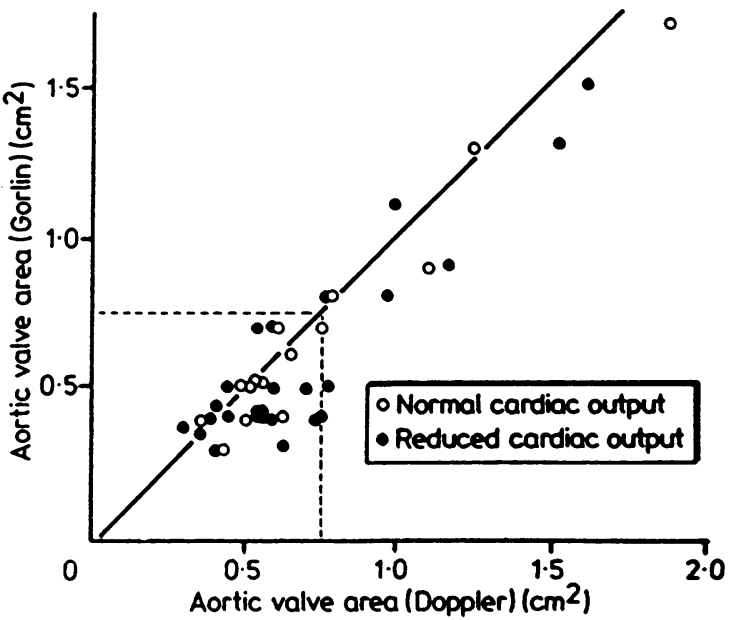

Fig 5 Plot of Doppler and invasive measurements of the aortic valve area showing line of equality. A cardiac index of $<3 \mathrm{l} / \mathrm{min} / \mathrm{m}^{2}$ was regarded as indicating a decreased cardiac output. The linear correlation coefficient was 0.94. It was 0.91 when cardiac output was reduced and 0.96 when it was normal. The area within the dotted lines corresponds to critical areas $\leqslant 0.75 \mathrm{~cm}^{2}$.
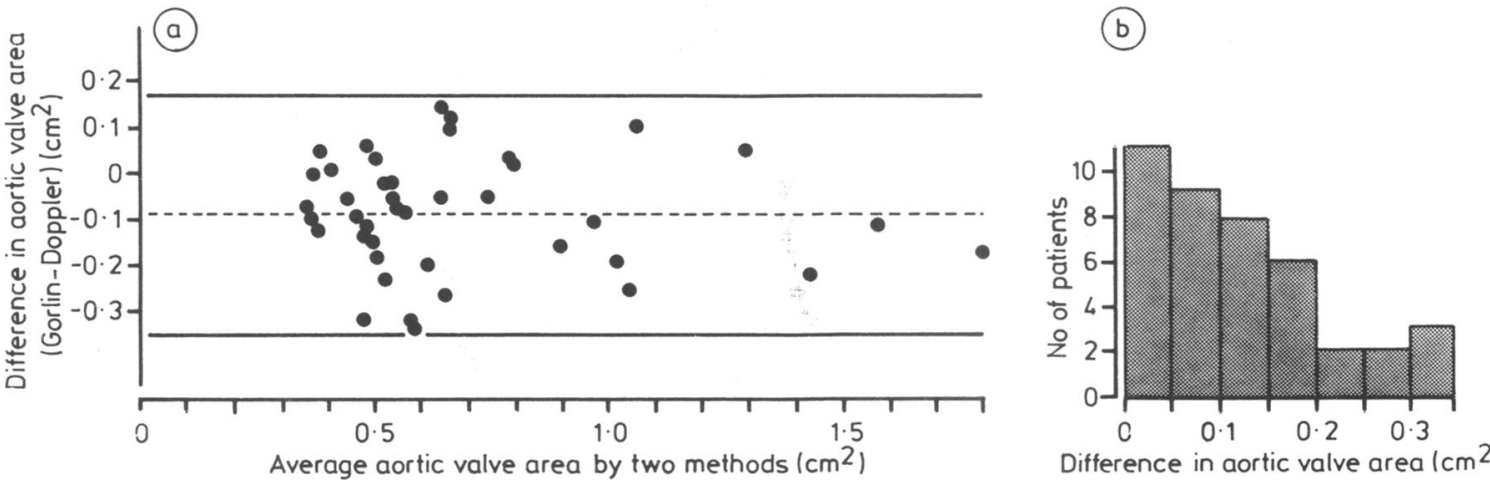

Fig 6 Differences between non-invasive and invasive measurements of the aortic valve area. (a) Differences plotted against mean value for both measurements (horizontal axis). Dotted line represents the mean value of the differences. The limits of agreement are shown. The standard error of the mean was $0.02 \mathrm{~cm}^{2}$. (b) Absolute values of the differences between both measurements are shown on the abscissa and the number of patients with a particular value is shown on the ordinate. 
findings were characterised by the absence of an audiosignal of systolic flow over the aortic orifice, except for a restricted area where there was a harsh tone. The corresponding graphic display showed a broadened spectrum over the first two thirds of systole, with a mid-systolic window in some cases. Velocity signals due to cusp motion could also be heard. The narrowed area was generally round (23) or ovoid with a major anteroposterior (5) or transverse (6) axis. More rarely it was triangular (4) or a transverse slit (3). It was located at the centre of the orifice in 18 patients and eccentrically in 23 patients. Doppler prediction of the site of the narrowed orifice was confirmed in nine patients at operation (six central and three eccentric locations). Two patients showing a slit like central area at Doppler examination were found to have a Monckeberg sclerosis without commissural fusion. In the remaining patients who came to operation cor- relation between the Doppler findings and direct examination was made impossible by conspicuous. calcifications. Table 1 shows individual and mean values of haemodynamic and Doppler data. Only one patient (case 25) was erroneously classified as -

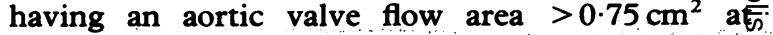
Doppler. The mean value of the aortic valve area was larger (NS) at Doppler than at catheterisation 0 Figure 5 shows the values of the measurements with? the line of equality. The mean difference was. $-0.09 \mathrm{~cm}^{2}$ with a standard deviation of 0.13 . The limits of agreement ranged from -0.35 tow $+0.17 \mathrm{~cm}^{2}$ (fig 6a). Figure $6 \mathrm{~b}$ shows that in 265 patients the difference did not exceed $0 \cdot 10 \mathrm{~cm}^{2}$. The greatest differences were $0.33,0.32,0.35 \mathrm{~cm}^{2}$ and these were found in patients with values of 0.4 (case $\vec{A}$ 16), 0.3 (case 21), and $0.4 \mathrm{~cm}^{2}$ (case 31 ) respectively by the Gorlin formula.

Figure 7 shows two observers' measurements with

Table Invasive determination of cardiac index and stenotic aortic valve areas by the Gorlin formula and the pulsed Doppler. mapping procedure in patients with aortic stenosis

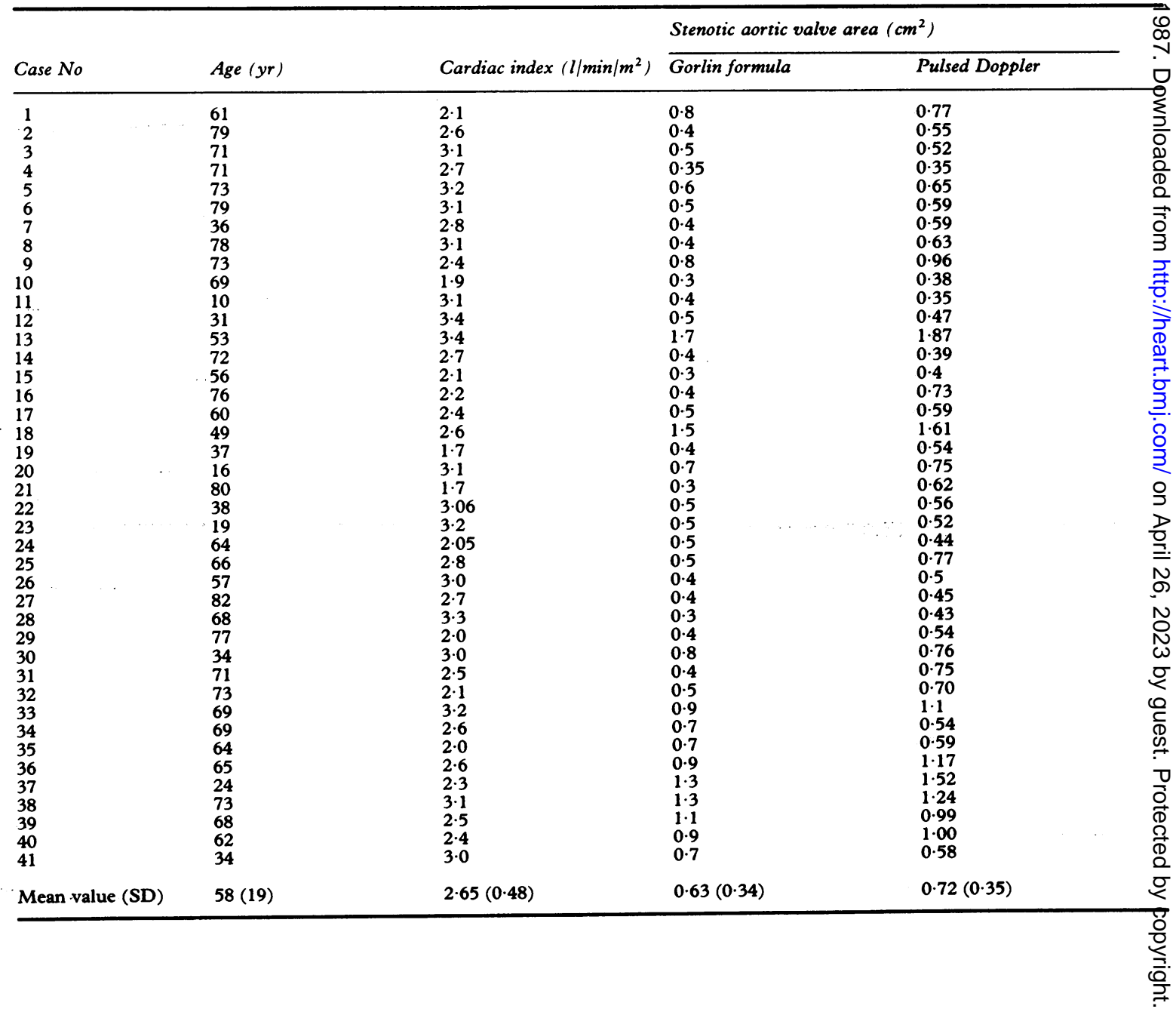




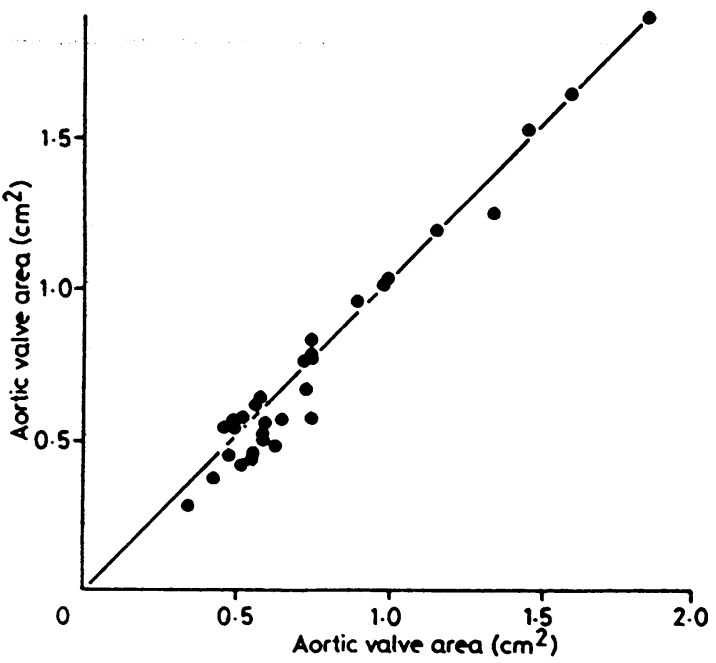

Fig 7 Measurements of the aortic valve area performed by two independent observers. There was good agreement between the measurements performed on 31 patients by two observers. The line of equality is shown. (SEM $0.01 \mathrm{~cm}^{2}$, correlation coefficient 0.95 ).

the line of equality. The mean difference was $0.02 \mathrm{~cm}^{2}$ with a standard deviation of 0.08 .

\section{Discussion}

In the present study we used mapping of the aortic valve area to quantify previously diagnosed aortic stenosis. The rationale of our method, which is based on measurements derived from the flow mapping procedure, has already been described and discussed for tricuspid ${ }^{19}$ and left sided regurgitation. ${ }^{20}$

Our results show that the Doppler mapping procedure provided a satisfactory assessment of the areas of flow. There was only one minor error of classification. Despite the good correlation between the invasive and the non-invasive data (fig 5), agreement between both methods was not complete; Doppler measurements often exceeded the values found at catheterisation. Figure $6 \mathrm{~b}$ shows that the differences were, however, often minimal, but with some instances of larger variation. Is this variability in the measurements clinically acceptable? When the severity of aortic stenosis is assessed the aim is to detect or exclude the need for operation-that is a flow area of $\leqslant 0.75 \mathrm{~cm}^{2}$. Figure 5 shows that the variability of the measurements did not affect the assessment of patients with large stenotic areas (close to or above $1 \mathrm{~cm}^{2}$ ). Nor did it affect the efficiency of the new method in detecting all but one of the critical areas despite very wide differences between the invasive and non-invasive measurements in three patients. In these three patients the invasive values were considerably less than
$0.75 \mathrm{~cm}^{2}$, which is often the case in severe stenosis. The range of areas $<0.75 \mathrm{~cm}^{2}$ and $>1 \mathrm{~cm}^{2}$ covers most clinical situations. When the size of a stenotic orifice lies close to the critical value of $0.75 \mathrm{~cm}^{2}$, however, the variability in measurements might lead to misclassification. In our study there was only one error of this type.

One explanation of the variability of the measurements may be insufficient spatial resolution of the sample volume. Spatial resolution varies with the depth of the recording site and with the size and shape of the sample volume. The width of the latter is determined by the beam width and the length by the pulse envelope. If the attenuation of the ultrasound intensity is limited by a factor of $2(6 \mathrm{DB})$ the sample volume will be an ellipsoid with a length of $1.5 \mathrm{~mm}$ (three sine waves of $3 \mathrm{MHz}$ ) and width of $1.5-2 \mathrm{~mm}$ (or stated more simply, a sphere of $2 \mathrm{~mm}$ diameter). ${ }^{21}$ The accuracy of the mapping procedure depends on the accuracy of the determination of the absence of flow at the edges of the area. This will be least accurate at positions between 2 and 3 (fig 8); theoretically this error will be very small because of the decreased energy of the $3 \mathrm{MHz}$ ultrasound wave reflected from both these positions. In most cases these errors are likely to be reduced by other factors, mainly the high pass filters of the velocimeter and the well known sharp decrease of the jet velocity profile within $0.5 \mathrm{~mm}$ to $1 \mathrm{~mm}$ of the edges. In some cases, however, the determination of the limits of flow might be affected by the extension of the boundary layer over a wider cross section of the jet than usual, as was shown experimentally in tight stenoses. ${ }^{22}$ This could lead to inaccuracy and might explain the scattered values for critical areas within the $0.75 \mathrm{~cm}^{2}$ limits on fig 5 .

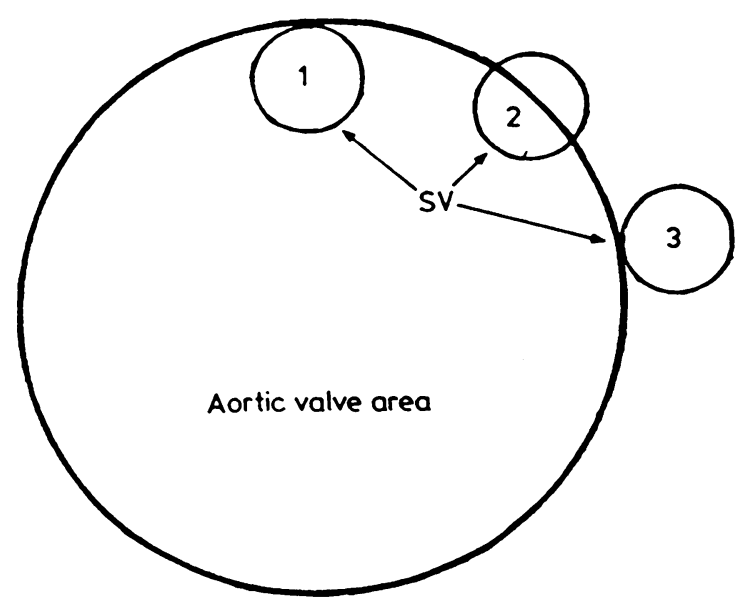

Fig 8 Schematic representation of the different positions of the sample volume along the border of the flow area. See text for explanation. SV, sample volume. 
Two other causes of variability between Doppler and invasive measurements should also be kept in mind. Firstly, the plane of the Doppler examination may differ slightly from that at which the jet starts. This is most unlikely to have occurred in our study because of the high correlation found between the measurements of the anteroposterior axis of the flow area obtained from short and long axis views. The long axis view ensured that the measurement was performed at the tips of the aortic valves. Secondly, the Gorlin formula produces an average figure by unit of time, representing a functional value of the area, whereas the Doppler measurements correspond to the area of instantaneous flow passing through an anatomical orifice. In addition, the Gorlin formula takes into account the contraction coefficient of the jet after its starting point; this may account for some of the observed differences between both values. Surgical or pathological correlation was available in only a few cases. The relatively high percentage $(44 \%)$ of asymmetrical orifices shows that caution is required in using a single diameter to calculate the true area.

As with all ultrasonic procedures a poor signal to noise ratio may limit the clinical application but future technological improvements should improve performance. Although the newer flow mapping multigate devices should make the procedure easier, they will also be affected by the problem of spatial resolution. The presence of low cardiac output is not a limiting factor because the new procedure remained accurate throughout the clinical range.

This work was partly supported by grants from CNAMTS and the ARNTIC Research Association.

\section{References}

1 Benchimol A, Dimond EG, Shen Y. Ejection time in aortic stenosis and mitral stenosis. Am $\mathcal{F}$ Cardiol 1980;5:728-39.

2 Weyman AE, Feigenbaum H, Dillon JC, Chang S. Cross sectional echocardiography is assessing the severity of valvular aortic stenosis. Circulation 1975;52:828-34.

3 DeMaria AN, Bommer W, Hoye J, Lee G, Bouteller J, Mason DT. Value and limitations of cross sectional echocardiography of the aortic valve in the diagnosis and quantification of valvular aortic stenosis. Circulation 1980;62:304-12.

4 Hatle L, Angelsen BA, Tromsdal A. Non-invasive assessment of aortic stenosis by Doppler ultrasound. Br Heart $\mathcal{F}$ 1980;43:284-92.

5 Stamm RP, Martin RP. Quantification of pressure gradients across stenotic valves by Doppler ultrasound. f Am Coll Cardiol 1983;2:707-18.

6 Hoffmann A, Burckhardt D. Evaluation of systolic murmurs by Doppler ultrasonography. Br Heart $\mathfrak{f}$ 1983;50:337-42.

7 Kosturakis D, Allen HD, Goldberg SJ, Sahn DJ,
Valdes Cruz LM. Non invasive quantification of ste- $\frac{\varrho}{\mathrm{C}}$ notic semilunar valve areas by Doppler echo- $C$. cardiography. F Am Coll Cardiol 1984;3:1256-62.

8 Griffith J, Henry WL. An ultrasound system for car- $\stackrel{\infty}{+}$ diac imaging and Doppler blood flow measurement $\mathrm{C}$ in man. Circulation 1978;56:925-30.

9 Goldberg SJ, Sahn DJ, Allen HD, Valdes Cruz LM, Hoenecke H, Carnahan Y. Evaluation of pulmonary and systemic blood flow by two-dimensionalo Doppler echocardiography using fast Fourier transform spectral analysis. $A m$ f Cardiol $\overrightarrow{0}$ 1982;50:1394-400.

10 Warth DC, Stewart WJ, Block PC, Weyman AE. A $\vec{\sigma}$ new method to calculate aortic valve area without left heart catheterisation. Circulation 1984;70:978-83.

11 Gorlin R, Gorlin SG. Hydraulic formula for calcu- or lation of the area of the stenotic mitral valve, other- cardiac valves and central circulatory shunts. $A m+\dot{+}$

Heart f $1951 ; 41: 1-29$.
12 Carrillo Kabana J, Calderon Montero J, Lopez Sendon J, Fuertes Garcia A, Pey-Illera J. Qualitative and ${ }_{c}^{-}$ quantitative evaluation of aortic and mitral insufficiencies. Comparative study. Rev Esp Cardiole 1976;29:227-35.

13 Johnson SL, Baker DW, Lute R, Dodge HT. Doppler echocardiography. The localization of cardiac mur- $\infty$ murs. Circulation 1973;48:810-27.

14 MacPherson PC, Meldrum SJ, Tundstall Pedoe DS. Angioscan: a spectrum analyser for use with ultrasonic Doppler velocimeters. F Med Eng Technol 1981;5:84-5.

15 Spencer MP, Fujioka K. CW Doppler with spectrafo analysis in acquired valve disease. In: Spencer $M D, \varrho$ ed. Cardiac Doppler diagnosis. Boston: Martinus $\overline{\bar{\sigma}}$ Nijhoff, 1983:153-74.

16 Veyrat C, Kalmanson D, Farjon M, Guichard JP, Sainte Beuve D, Abitbol G. Combined pulsed. Doppler echocardiography for the investigation of valvular heart diseases: one dimensional versus two dimensional approach. In: Rijsterborgh $\mathrm{H}$, ed. Echocardiology. Boston: Martinus Nijhoff, 1981:291-8. 후

17 Nishimura K, Sakakibara T, Hibi N, et al. High? speed ultrasonocardiotomography: echocardiographic manifestations of papillary muscles andB chordae tendineae. $\mathcal{F}$ Cardiogr 1977;7:1-13.

18 Bland JM, Altman DG. Statistical method for assessing agreement between two methods of clinical measurement. Lancet 1986; i:307-10.

19 Miyatake K, Okamoto M, Kinoshita N, et al. Evalu N ation of tricuspid regurgitation by pulsed Doppler and two dimensional echocardiography. Circulation 1982;66:777-84.

20 Veyrat C, Abitbol G, Bas S, Manin JP, Kalmanson Dס Quantitative assessment of valvular regurgitatio using the pulsed Doppler technique. Approach to the regurgitant lesion. Ultrasound Med Biof 1984;10:201-13.

21 Jorgensen JE, Campau DN, Baker DW. Physical char acteristics and mathematical modelling of the pulse ultrasonic flowmeter. Med Biol Eng 1973;11:404-21?

22 Holen J. Quantification of flow obstruction in mitraß stenosis and prosthetic mitral valves with Dopplev ultrasound. In: Spencer MP, ed. Cardiac Dopplek diagnosis. Boston: Martinus Nijhoff, 1983:337-42. 음 